\title{
GEOMORPHOLOGICAL MAPPING WITH TERRESTRIAL LASER SCANNING AND UAV-BASED IMAGING
}

\author{
N. Tilly ${ }^{a, *}$, D. Kelterbaum ${ }^{\text {a }}$, R. Zeese ${ }^{a}$ \\ ${ }^{a}$ Institute of Geography, University of Cologne, 50923 Cologne, Germany - \\ (nora.tilly, daniel.kelterbaum)@uni-koeln.de, reinhard.zeese@web.de
}

\begin{abstract}
KEY WORDS: Terrestrial laser scanning, UAV-based imaging, 3D data, Digital elevation model, High-resolution mapping, Karst landscape, Surface monitoring
\end{abstract}

\begin{abstract}
High-resolution digital elevation models (DEMs) are useful for the detailed mapping of geomorphological features. Nowadays various sensors and platforms are available to collect 3D data. The presented study compares terrestrial laser scanning (TLS) and low-cost unmanned aerial vehicles (UAV)-based imaging in terms of their usability for capturing small-scale surface structures. In October 2014 and June 2015 measurements with both systems were carried out in an episodically water-filled karst depression under pasture farming in the region of Hohenlohe (Southwest Germany). The overall aims were to establish high-resolution DEMs and monitor changes of the relief caused by dissolution and compare the advantages and drawbacks of both systems for such studies. Due to the short time between the campaigns the clear detection of temporal changes was hardly possible. However, the multi-temporal campaigns allowed an extensive investigation of the usability of both sensors under different environmental conditions. In addition to the remote sensing measurements, the coordinates of several positions in the study area were measured with a RTK-DGPS system as independent reference data sets in both campaigns. The TLS- and UAV-derived DEM heights at these positions were validated against the DGPS-derived heights. The accuracy of the TLS-derived values is supported by low mean differences between TLS and DGPS measurements while the UAV-derived models show a weaker performance. In the future years additional simultaneous measurements with both approaches under more similar vegetation conditions are necessary to detect surface movements. Moreover, by investigating the subsurface the interaction of above and below ground processes might be detected.
\end{abstract}

\section{INTRODUCTION}

The purpose and content of a geomorphological map are an almost area-wide presentation of terrain features in a sufficiently high topographic and objective differentiation (Zepp, 2014). This can involve characteristics of the relief and the shallow subsurface but also the morphogenesis and morphodynamic of an area. Which degree of detail can or should be reached, highly depends on the research question and on the available measuring methods. Historically, the most common acquisition method is the manual drawing with pen and paper. Guidelines for geomorphological mapping (Leser and Stäblein, 1975) ensure thereby the comprehensibility and comparability of maps drawn by different authors. The objectivity and accuracy of measurements can be enhanced by simple technical devices, such as a compass, a barometer, or an inclinometer. Nevertheless, the detailedness of such maps is always limited and the quality highly depends on the drawer. Hence, almost autonomic methods are desirable for an unbiased and objective acquisition.

\subsection{Remote sensing for geomorphological mapping}

In the past several decades the importance of remote sensing for studies on the natural environment increased (Jensen, 2007). Applications range from studies on vegetation and water to the assessment of soils, minerals, or geomorphological structures. In particular for the latter, the detection of 3D structures is a major topic. A widely applied technology therefore are measurements based on Light Detection and Ranging (LiDAR). Depending on the regarded area of interest and targeted resolution, LiDAR measurements can be performed as airborne laser scanning (ALS) or terrestrial laser scanning (TLS). The usability of both methods is well documented (Buckley et al., 2008; Tarolli, 2014).
Although TLS measurements are considerably cheaper and easier to perform than ALS approaches, the effort and expense should still not be underestimated.

Over the last few years, the development and widespread availability of unmanned aerial vehicles (UAV) and increasing quality of affordable conventional digital cameras induced a growing research focus on the usability of such systems for scientific studies (Colomina and Molina, 2014; Shahbazi et al., 2015). One main focus is to be as flexible and cost-effective as possible. A common way to generate $3 \mathrm{D}$ information from the captured images are approaches based on structure from motion (SfM) (Turner et al., 2012; Westoby et al., 2012). As such approaches are however quite young and little research has been done so far on the accuracy and reliability of collected data, comparative studies with trustworthy data and measuring procedures are necessary. Tong et al. (2015) investigated the integration of UAV-based photogrammetry and TLS for the mapping of open-pit mine areas. Advantages and disadvantages of UAV-based approaches in physical geography were compared to TLS by Smith et al. (2015) and Ouédraogo et al. (2014).

Nevertheless, only a few studies on the practical application and comparison of simultaneously acquired data sets exists. The presented study is based on a multi-temporal data set taken in an episodically water-filled karst depression. In each of two campaigns the area was captured with TLS and a UAV-based imaging system. While TLS can be regarded as reliable approach but requiring fairly high effort and costs, the UAV-based approach aims to be simple to perform and cost-efficient. The main focuses were: (I) to investigate the usability of both systems in the field, (II) to compare the achieved data campaign-wise, (III) to examine the capability of both approaches for detecting

\footnotetext{
* Corresponding author: nora.tilly@uni-koeln.de
} 


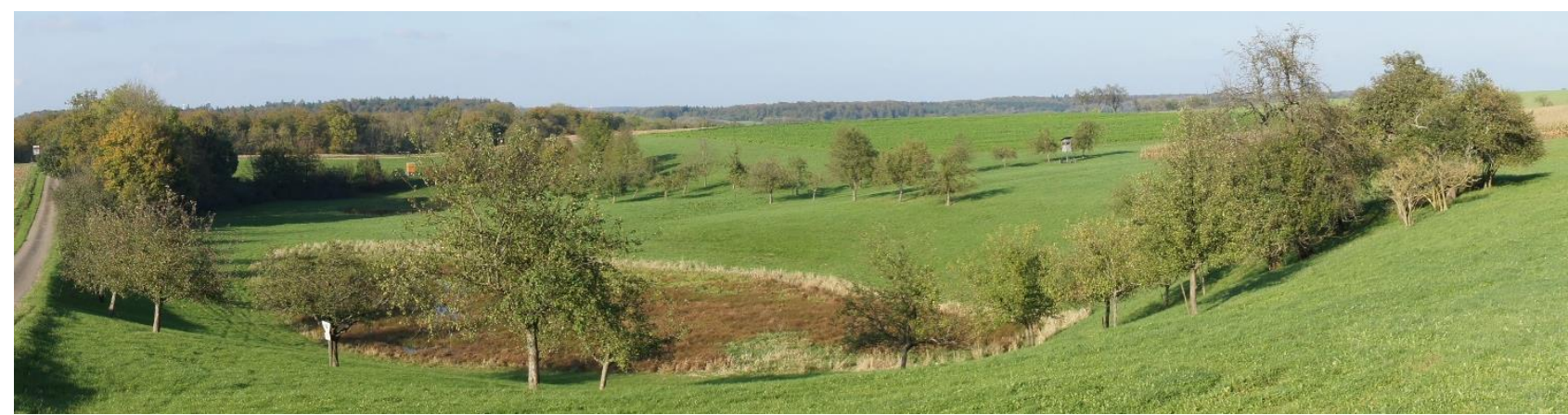

Figure 1. Karst depression (Ottensee) in the region of Hohenlohe, Baden-Württemberg. Picture captured from the south side.

temporal changes, and (IV) to evaluate the measured height values against reference measurements.

\subsection{Study area}

During the Triassic ( 230 to 195 million BP; Ahnert, 2009), the today's central Europe was situated in the subtropics and sediments were accumulated under marine environs. During this time calcareous sediments, shells, and other marine features were deposited and fossilized which is also indicated by the name of this lithostratigraphic unit Muschelkalk (shellbearing limestone). It covers the older Buntsandstein (colorful sandstone) and lies below the younger Keuper. Outcrops can be found in Middle and South Germany, where weathering of the limestone induces the development of karstic phenomena. Such regions are marked by the appearance of sinkholes, ponors, or karst depressions for example (Zepp, 2014).

For this study, an episodically water-filled karst depression (Ottensee, Figure 1) in the region of Hohenlohe was investigated (German federal state Baden-Württemberg, N 49²1' 17", E $9^{\circ}$ 49' 06"). Situated in a larger valley, the area of about $100 \mathrm{~m}$ by $200 \mathrm{~m}$ in size is mainly used for pasture farming during almost the entire year. However, after torrential rain the subterranean run-off in higher lying aquiferous layers can cause a flooding of the depression, resulting in a small lake. Depending on the filling level the water drains off again within hours or a few days through a swallow hole at the lower end of the depression in the northern part. This episodic flooding is however likely to change the surface and to induce dissolution processes in the subsurface.

\section{METHODS}

Two field campaigns were carried out in October 2014 and June 2015. In each campaign the area was captured with a TLS system and a UAV-based imaging system. Each data set was used to establish high-resolution digital elevation models (DEMs). This allowed both, a comparison between the multi-temporal data sets of each sensor and a comparison between the resulting DEMs of both sensors under different vegetation conditions. In addition, several points were measured with a RTK-DGPS system in each campaign as reference data set. In Table 1 , all dates of the TLS and UAV campaigns are listed. Moreover, the DGPS-derived point measurements are categorized in set A - D.

\begin{tabular}{lll}
\hline Date & Data source & Data set \\
\hline 24.10 .14 & TLS-derived point cloud & DEM \\
& DGPS_1 \\
25.10 .14 & UAV-derived points & set $(\mathrm{n}=17)$ \\
& DGPS-derived points & DEM $_{\text {UAV_1 }}$ \\
set $\mathrm{B}(\mathrm{n}=21)$ \\
04.06 .15 & TLS-derived point cloud & DEM $_{\text {TLS_2 }}$ \\
& DGPS-derived points & set $\mathrm{C}(\mathrm{n}=38)$ \\
07.06 .15 & UAV-derived orthophoto & DEMUA__2 \\
& DGPS-derived points & set $\mathrm{D}(\mathrm{n}=19)$ \\
\hline
\end{tabular}

Table 1. Acquired data sets.

\subsection{Terrestrial laser scanning}

The area was scanned with the terrestrial laser scanner Riegl LMS-Z420i (Riegl LMS GmbH, 2010). The digital camera Nikon D200 is mounted on the laser scanner. Point clouds gained from the laser scanner can be colorized from the recorded RGB-images. This time-of-flight scanner operates with a near-infrared laser beam, the beam divergence is $0.25 \mathrm{mrad}$, and it can measure up to 11,000 points/sec. Its field of view is up to $80^{\circ}$ in the vertical and $360^{\circ}$ in the horizontal direction. In this study a resolution of $0.04^{\circ}$ was chosen. Six to eight scan positions were established in each campaign for covering the whole area. The coordinates of the scan positions and additional targets were measured with the highly accurate RTK-DGPS system Topcon HiPer Pro (Topcon Positioning Systems, 2006). These information were used in the post-processing for the georeferencing and co-registration of both the different scan positions of one campaign and the data sets of both campaigns.

In the post-processing, the scanner software RiSCAN Pro was applied for merging, cleaning, and extracting the area of interest (AOI). Afterwards a software implemented terrain filter was used to remove most of the vegetation. However, an additional manual editing could not be prevented. After importing the final point clouds to Esri ArcGIS Desktop 10.2.1 a raster data set containing the DEM height values was created using the inverse distance weighting (IDW) algorithm.

\subsection{Airborne imaging}

Low-cost UAV-based imaging was performed with a GoPro Hero 3+ (GoPro, 2015) attached under a DJI Phantom 2 (DJI, 2015). In each campaign about 300 images were acquired. For the georeferencing, about 20 ground control points (GCPs) were measured with the Topcon HiPer Pro RTK-DGPS system (Set B and D). From the UAV-derived images orthophotos were calculated and the DEMs were generated with the SfM-based software Agisoft PhotoScan 1.1.3. 


\subsection{Comparison and temporal development}

Due to the different acquisition methods and times of recording the resulting point density and distribution was quite heterogeneous in the AOI. Moreover, the respective status of the vegetation had a significant influence on the measurements. As shown in the top of Figure 2, the pasture cover in the AOI was quite flat in the first campaign, since it was conducted in autumn. The second campaign had been scheduled for early summer to have a higher probability of good weather for simultaneous drillings in the karst depression. Unfortunately, mowing the pasture was only possible several hours before the TLS measurements due to continuous rain during the previous days. Since the cuttings were not collected by the lawnmower, they remained as rows of cut grass in the AOI as shown in the bottom of Figure 2. As it was with both systems impossible to penetrate these layers a temporal comparison of highly resolved DEMs was not meaningful. Hence, the TLS- and UAV-derived raster data sets were resampled to a common resolution of $0.20 \mathrm{~m}$, which can still be regarded as being a sufficiently high resolution for this study. With the raster calculator in ArcGIS, these comparable DEMs were then used to calculate the difference between the those of each campaign but generated from different sensors (e.g. DEM $M_{U A V}{ }_{1} 1$ minus DEMTLS_1) and the difference between the models of one sensor but from the different campaigns (e.g. DEMTLS_1 minus DEMTLS_2).

\subsection{Evaluation}

The DGPS-derived reference data sets were used for evaluating the DEMs. All point coordinates were converted to vector point data for automatically extracting the height values from the DEMs. According to their acquisition time, set $\mathrm{A}$ and $\mathrm{B}$ were used for evaluating DEMTLS_1 and DEMUAv_1, and set C and D for DEMTLS_2 and DEMUAv_2. It has to be mentioned that set B and $\mathrm{D}$ were already used for the georeferencing of the respective UAV-derived images. Hence, these data sets are only a completely independent reference for the TLS-derived DEMs. In a created buffer area with a radius of $0.50 \mathrm{~m}$ around each point, the lowest values of the respective DEM were extracted. These values were used to prevent overestimations due to remaining vegetation in the calculated DEMs.

\section{RESULTS}

According to the multi-temporal and multi-sensor approach quite extensive results were achieved. The following primarily presents the four established DEMs and their main characteristics. Then the differences between DEMTLS_1 and DEMuAv_1 are listed as a comparison of both sensors and a limited attempt is made to analyze the temporal development.

\subsection{Digital elevation models}

The TLS- and UAV-derived DEMs were resampled to a common resolution of $0.20 \mathrm{~m}$. Maps of the four resulting DEMs are shown in Figure 2. Overall, the main characteristics can be summarized as follows:

- In all DEMs, an almost permanently water-filled part in the southern part of the AOI with higher grass and reeds (also visible in the foreground of Figure 1) is identifiable.

- Both TLS-derived DEMs show a finer spatial differentiation than the UAV-derived ones.

- As the UAV-derived data were not cleaned from vegetation, the height values are overall slightly higher and quite high values can be related to trees at the edges of the AOI.

- Both UAV-derived DEMs show fairly low values in the northern part, which suggests a systematic error in the UAVbased approach.

- The rows of cut grass are detectable in the DEMs of June, in particular in the TLS-derived model and sharp edges between the mown and unmown parts are visible.

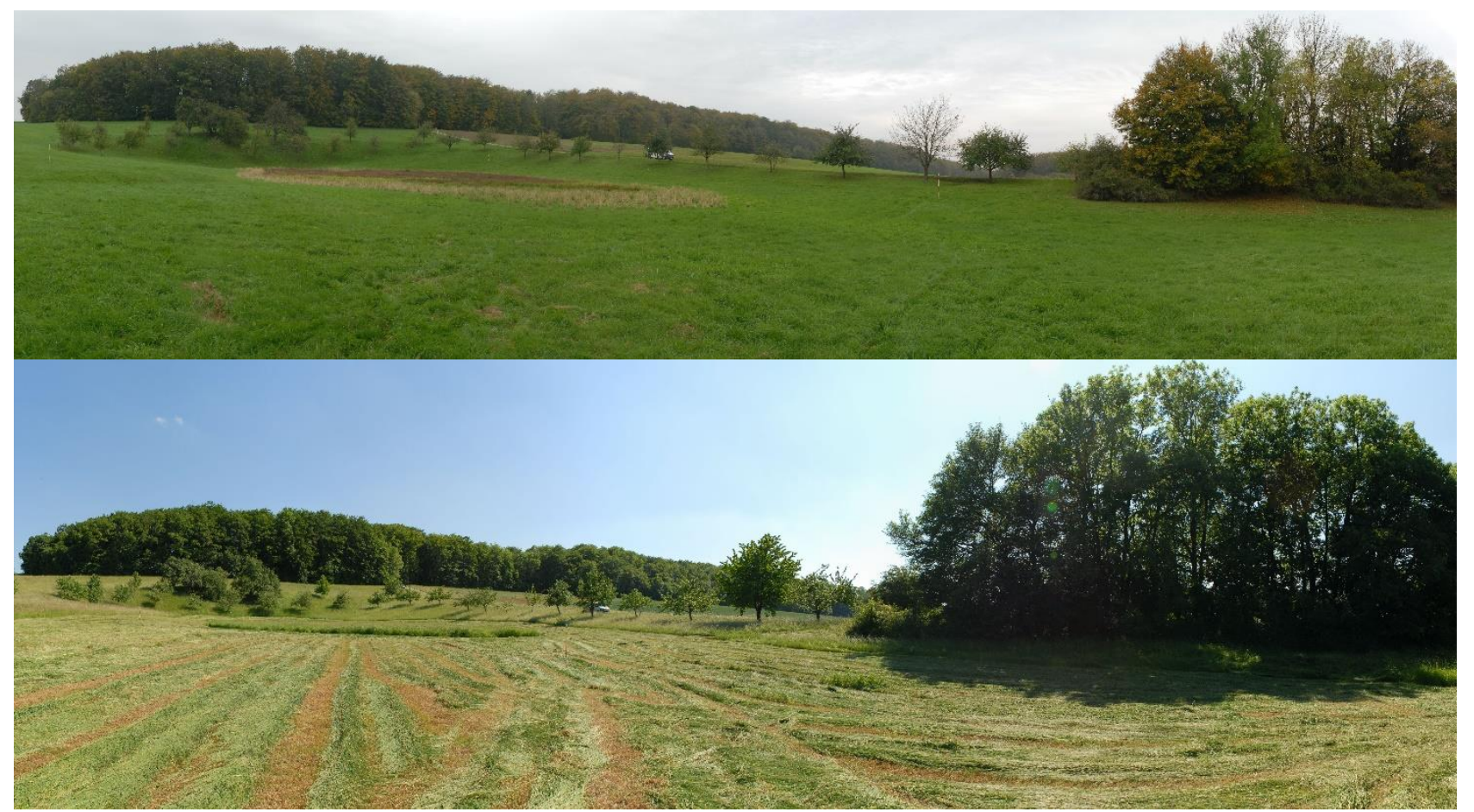

Figure 2. AOI captured with the camera mounted on the laser scanner at a scan position in the middle of the karst depression, viewing towards southwest, in October 2014 (top) and June 2015 (bottom). 


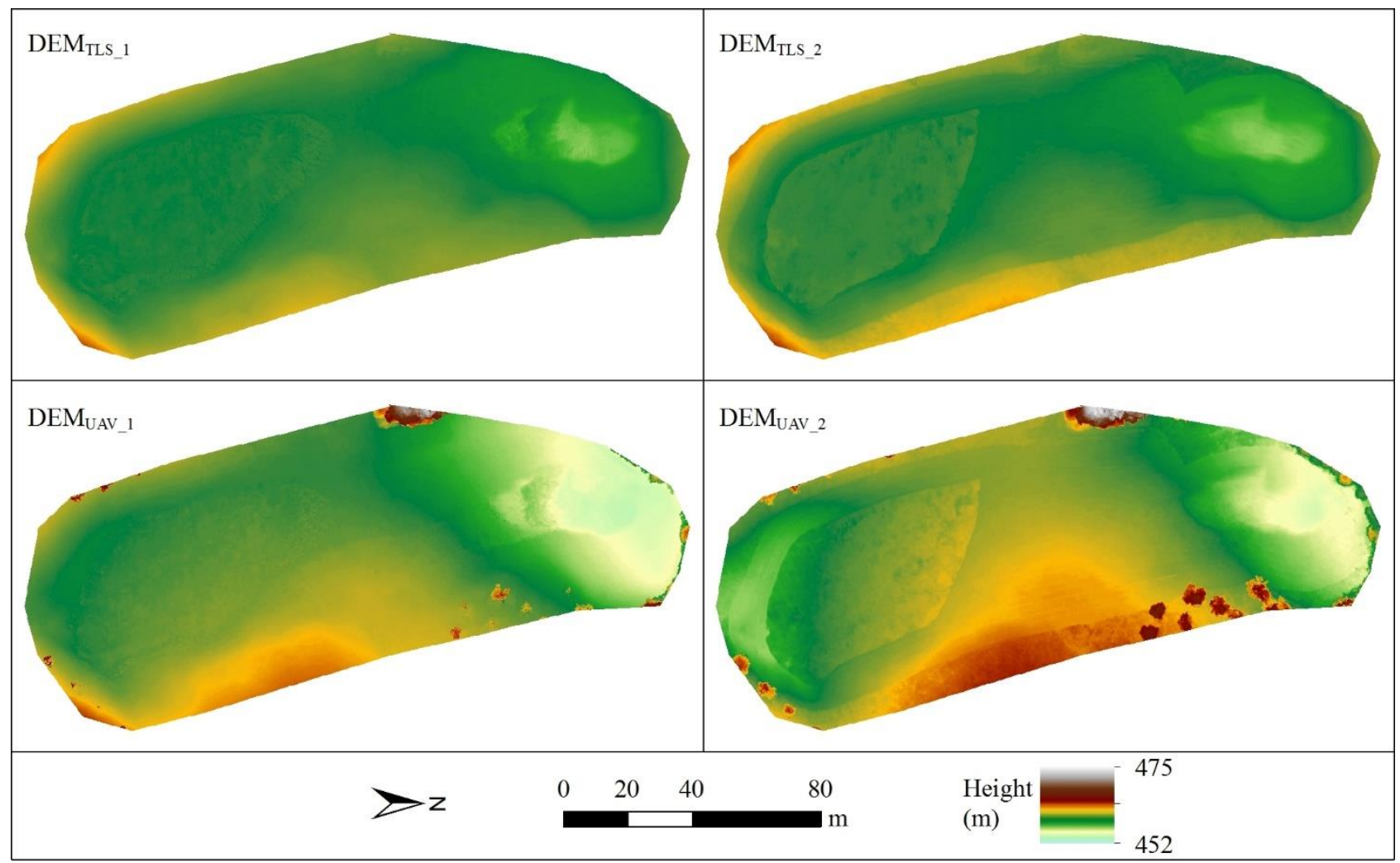

Figure 3. TLS-derived (top) and UAV-derived DEMs (bottom), each from October 2014 (left) and June 2015 (right).

\subsection{Sensor comparison}

The difference between the DEMs of one campaign but derived from the different sensors was calculated to compare the resulting DEMs. Figure 4 shows this for the data recorded in October. The DEM $_{\text {TLS_1 }}$ was therefore subtracted from DEMUAv_1. Although the mean difference between both raster data sets is small with $0.04 \mathrm{~m}$, the standard deviation is quite large $(1.56 \mathrm{~m})$. Moreover, discernible spatial patterns are observable in Figure 4. In the northern half of the karst depression and with increasing values northwards, the difference is negative, hence the UAV-derived elevation values are lower than the TLS-derived ones. Outgoing from a semicircular area at the southeast edge the difference is positive with decreasing values towards the remaining parts of the depression. The small irregular formed areas with high positive values are a result of the trees and bushes which were not removed from the UAV-derived data.

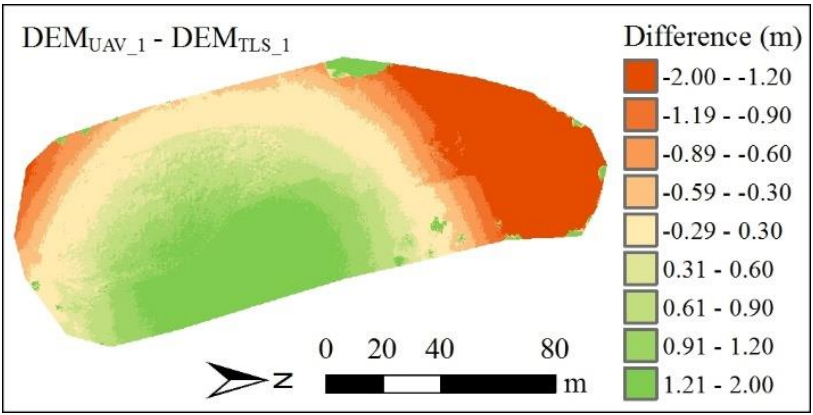

Figure 4. Difference between TLS- and UAV derived DEMs recorded in October 2014 (mean $=0.04 \mathrm{~m}$; stand. dev. $=1.56 \mathrm{~m})$.

\subsection{Temporal development}

Beside the evaluation of the two measuring approaches, the initial question for this project was to check whether the episodic filling with water induces a change of the relief in the karst depression. In order to investigate this development, the DEM $\mathrm{TLS}_{\_} 2$ was subtracted from DEMTLS_1 (Figure 5 top) and DEMuAv_2 from DEMUAv_1 (Figure 5 bottom). Since the DEMs captured in October were subtracted from the DEMs in June, positive values (greenish color) indicate increasing elevation heights and negative values (reddish color) show decreasing elevation heights.

Overall, very different results were achieved with both approaches. The difference between the TLS-derived DEMs is with lower than $\pm 0.20 \mathrm{~m}$ quite small in most parts of the karst depression. Strongly negative values occur in a small area in the middle of the northern part, which are explainable through the removal of blackberry bushes preparatory to the campaign in June. Highly positive values occur at the edges of the AOI and in the almost permanently water-filled area in the southern part. These differences are clearly explainable through the uncut grass in these parts. Nevertheless, the highest positive values are findable in a small part at the south edge of the almost permanently water-filled area. At this position the spring, where the subterraneous water comes to the surface is located. It might thus be assumable that material transported with the water and deposited in this part could have caused the rising elevation values. However, the value of $0.60 \mathrm{~m}$ and more is quite high for an accumulation of sediments of this shot time interval. This absolute value might thus be a sum of sediment accumulation and a higher vegetation. 


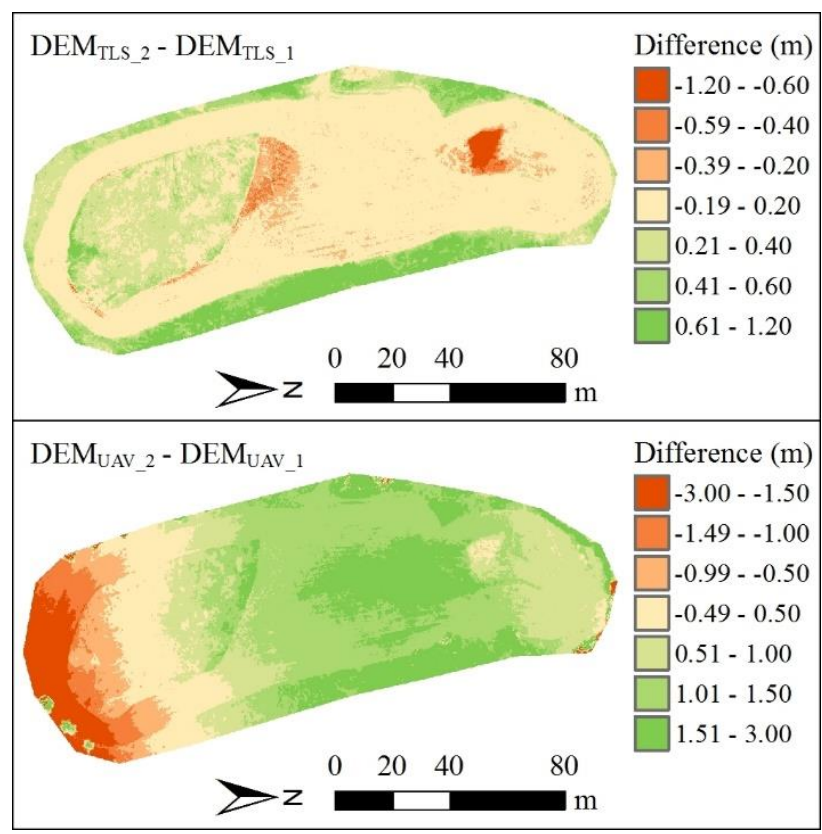

Figure 5. Difference between DEMs recorded in October 2014 and June 2015 from TLS-derived (top) and UAV-

derived (bottom) data.

Regarding the UAV-derived DEMs, a systematic error is quite obvious. Even though a visual comparison of the DEMs shown in Figure 3 would suggest that they are quite similar, the calculated difference reveals something else. Strongly negative values occur at the south edge, which increase northwards. Hence, it very likely that the DEMs are somehow tilted against each other. Unfortunately, this error could not be solved, which made a further analysis of the temporal development based on the UAV-derived data not meaningful.

\subsection{Evaluation}

As reliable reference data, the point measurements from the RTK-DGPS were used. The main statistics of this quality analysis are shown in Table 2. Considering their acquisition time, set $\mathrm{A}$ and $\mathrm{B}$ were used for evaluating the DEMs captured in October 2014 and set C and D for the DEMs captured in June 2015. Overall the results for the evaluation of the TLS-derived DEMs are much better than the UAV-derived ones. The mean difference and standard deviation between DGPS- and TLS-derived data is with always both lower than $\pm 0.10 \mathrm{~m}$ very small and almost similar for each point set. It has to be noted that the values of the TLS-derived DEMs are averaged across buffer area with a radius of $0.5 \mathrm{~m}$, hence minor variations must be assumed. The mean differences and standard deviations between DGPS- and UAV-derived elevation heights are generally higher. Better results were achieved for the DEMUAv_2. Moreover, lower values were reached with set $\mathrm{B}$ and $\mathrm{D}$ than with $\mathrm{A}$ and $\mathrm{C}$. However, since these points were already used as GCPs during the DEM generation, the sets cannot be regarded as completely independent reference.

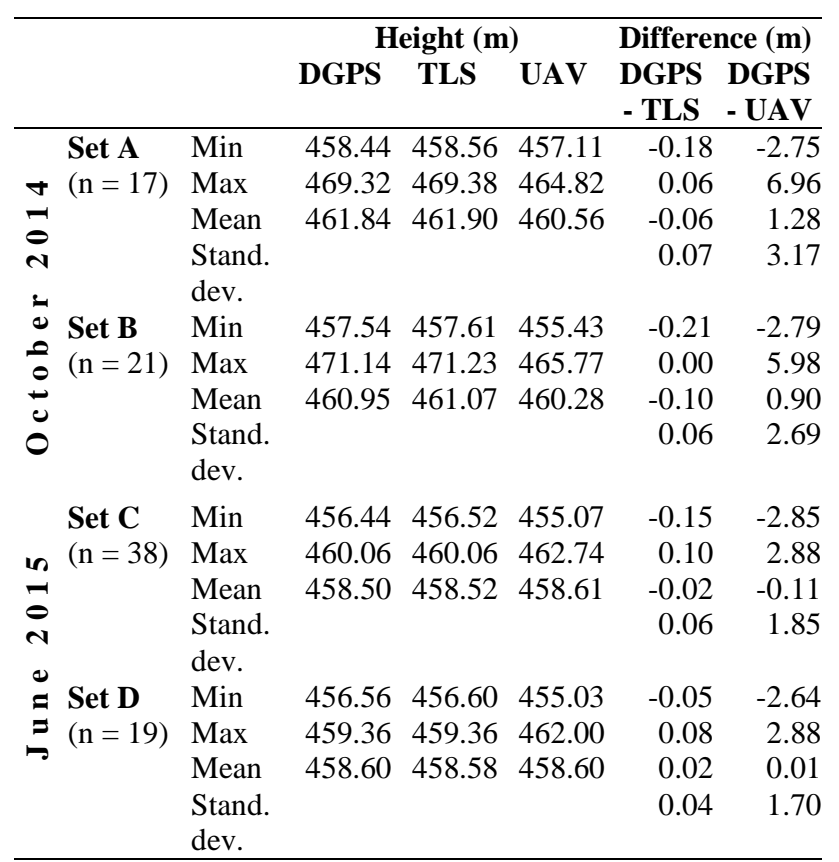

Table 2. Quality analysis of the DEMs, compared to DGPS reference measurements. Set A and B were used for DEMTLS_1 and DEMUAv_1; set C and D for DEMTLS_2 and DEMUAv_2.

\section{DISCUSSION}

In this study a multi-temporal and multi-sensor data set was investigated regarding the applicability for geomorphological mapping and surveying. The main objectives were (I) to check the usability of TLS and UAV-based imaging for such studies under field conditions, (II) to compare the achieved data, (III) to investigate whether temporal changes can be detected, and (IV) to validate the measured height values against reference measurements.

Both approaches were practicable in the field and are thus likely to be feasible for geomorphological mapping. Advantages and disadvantages have thereby to be stated for each system. The laser scanner as ground-based active system is more robust against poor weather but more difficult to transport, since it is heavier than the UAV-based imaging system. This is particularly important since it has to be carried manually between the scan positions. On the contrary, the UAV-based imaging system is very lightweight but prone to poor weather. Moreover, areas which are not or less accessible can hardly be capture with the scanner. In such cases UAV-based systems can be useful, which are however limited if dense trees obscure the nadir perspective.

Furthermore the required time has to be regarded. Due to the need of several scan positions, the manual transportation, and the measuring time, the TLS measurements were quite time consuming, with overall half a day in the field. Unfortunately, the herein used laser scanner was quite old. Recent trends in the development of laser scanning systems follow two directions. With the focus on longer scanning ranges, higher measuring rates, or a more lightweight buildup, the number of available high-end sensors increases, such as the Riegl VZ-2000 (Riegl LMS GmbH, 2015) or the Leica Scan Station P40 (Leica Geoystems, 2015). In contrast, the upcoming of cost-effective systems as the Velodyne HDL-64E LiDAR sensor (Velodyne, 2014) increase their availability for a broader audience. However, 
those systems mostly have a lower resolution. The developments are overall useful for accelerating the measuring process.

Coming back to the required time, the UAV-based imaging had the advantage that it could be performed within one hour and a half, including the placement and DGPS-measurement of the GCP positions. Moreover, measurements can generally be carried out very easy. Disadvantages of the used system were that flight paths have to be controlled manually with the Phantom 2 and the GoPro Hero 3+ has quite a low resolution. However, the UAV and camera markets are developing rapidly. Already with the Phantom 3 advanced (DJI, 2016) for example, waypoints can be predefined and hence flight paths can be predefined and controlled by an autopilot. The prices of conventional digital cameras decrease while the available sensor sizes and resolutions increase. Hence, rapid developments can be assumed in the near future.

The time requirements for the post-processing depend in particular on the expertise of the user and on the desired degree of detailedness. For example, in contrast to the TLS-derived point clouds, the UAV-derived data was not cleaned from vegetation. This step required some time but an unobscured representation of the relief can be expected then.

Concerning the achieved data, terrestrial laser scanners are known as trustworthy and accurate measuring devices which are numerously used for geomorphological studies (Buckley et al., 2008; Eltner and Baumgart, 2015; Tarolli, 2014). The herein generated DEMs show the suitability for capturing the relief of small-scale karstic forms such as the investigated karst depression. For an analysis of temporal changes more similar vegetation conditions are however favorable to prevent a blurring of the differences between the DEMs through the different vegetation heights. In this study the available filter options and downsampling to a common resolution allowed a first rough comparison of the DEMs. Nevertheless, further research is necessary and already initiated concerning filtering schemes based on the measured intensity value of the laser return (Guarnieri et al., 2009; Kashani et al., 2015).

Like in many other fields of research, UAV-based imaging approaches are increasingly used for geomorphological studies or more generally in physical geography (Favalli et al., 2012; Smith et al., 2015; Westoby et al., 2012). The chosen approach aims to be simple to perform and additionally cost-efficient. In this sense, the post-processing was reduced to a minimum. Even though the visual examination of the UAV-derived DEMs suggests the usability, the overall results were a bit too weak and not trustworthy enough to make a reliable statement. Better results can be expected from already scheduled further campaigns with a new and improved system.

Considering the temporal development, the short period of time between the two campaigns resulted in nearly undetectable surface movements. The acquisitions under different vegetation conditions allowed a consideration of the suitable time during the year for measurements in this context. As the vegetation produces noise in the point clouds and obscures the bare ground, measurements during the autumn or winter month are useful to capture relief features. However, the remote sensing measurements and most geophysical survey techniques for investigating the subsurface require good weather or at least dry conditions, which are more likely during spring and summer. Hence, an appropriate point in time is hardly determinable, but for investigating temporal changes similar environmental conditions are meaningful.
Finally, the validation against the RTK-DGPS reference measurements clearly demonstrated the better accuracy of the TLS data in comparison to the UAV-derived DEMs, which is also known from other studies (Ouédraogo et al., 2014). It can thus be summarized that the high quality of TLS measurements is necessary for capturing small-scale topographic features and their changes. Nevertheless UAV-based imaging offers a timeand cost-effective alternative for a rough acquisition of a terrain. As also suggested in other studies the combination or integration of laser scanning and photogrammetry should be a conceivable solution (Bates et al., 2008; Fabris et al., 2010; Nex and Rinaudo, 2011).

\section{CONCLUSION \& OUTLOOK}

From a technical point of view, one main aim of this study was to investigate the usability of a TLS system, as well-known and reliable device, and a UAV-based imaging system, as low-cost alternative, for the objective mapping of small-scale terrain features in geomorphological studies. In conclusion, both systems were easy to apply in the field while each sensor showed advantages and disadvantages. Main benefit of the TLS-data was the high degree of detailedness. In contrast the UAV-based imaging is much easier to perform.

With a more thematic focus, a further objective was to investigate whether the relief of the investigated karst depression showed a temporal development. However, with only about seven month the period of time between the two campaigns was quite likely too short for clearly noticeable changes. Moreover, the different vegetation conditions blurred the results. Changes of the surface close to the spring, where the subterraneous water comes to the surface might be detectable, but further campaigns under more similar vegetation conditions are necessary to verify this.

For the future, ongoing field campaigns with the laser scanner and a new and improved UAV-based system are planned for a multi-annual monitoring of the karst depression. Moreover, geophysical surveys are intended to investigate the subsurface and relate therein occurring changes to mass movements at the surface. Overall, the karst depression Ottensee offers an interesting study area for investigating the applicability of different remote sensing sensors for geomorphological mapping. The fairly easy accessibility and quite likely surface changes are thereby good preconditions.

\section{ACKNOWLEDGEMENTS}

We acknowledge the travel funding provided by the Graduate School of Geosciences (GSGS) of the University of Cologne (Grant No. GSGS-2016B-T02).

\section{REFERENCES}

Ahnert, F., 2009. Einführung in die Geomorphologie, 4th ed. UTB, Stuttgart.

Bates, K.T., Rarity, F., Manning, P.L., Hodgetts, D., Vila, B., Oms, O., Galobart, a., Gawthorpe, R.L., 2008. High-resolution LiDAR and photogrammetric survey of the Fumanya dinosaur tracksites (Catalonia): implications for the conservation and interpretation of geological heritage sites. J. Geol. Soc. London, 165, pp. 115-127. 
Buckley, S.J., Howell, J.A., Enge, H.D., Kurz, T.H., 2008. Terrestrial laser scanning in geology: data acquisition, processing and accuracy considerations. J. Geol. Soc. London. 165, 625638. Colomina, I., Molina, P., 2014. Unmanned aerial systems for photogrammetry and remote sensing: A review. ISPRS J. Photogramm. Remote Sens, 92, pp. 79-97.

DJI, 2016. Datasheet Phantom 3 http://www.dji.com/de/ product/phantom-3-adv

DJI, 2015. Datasheet Phantom 2 https://www.dji.com/ product/phantom-2

Eltner, A., Baumgart, P., 2015. Accuracy constraints of terrestrial Lidar data for soil erosion measurement: Application to a Mediterranean field plot. Geomorphology, 245, pp. 243-254.

Fabris, M., Baldi, P., Anzidei, M., Pesci, A., Bortoluzzi, G., Aliani, S., 2010. High resolution topographic model of Panarea Island by fusion of photogrammetric, lidar and bathymetric digital terrain models. Photogramm. Rec, 25, pp. 382-401.

Favalli, M., Fornaciai, A., Isola, I., Tarquini, S., Nannipieri, L., 2012. Multiview 3D reconstruction in geosciences. Comput. Geosci, 44, pp. 168-176.

GoPro, 2015. Datasheet GoPro Hero 3+ http://cbcdn2.gpstatic.com/uploads/product_manual/file/195/UM_H3PlusSilver _ENG_REVB_WEB.pdf

Guarnieri, A., Vettore, A., Pirotti, F., Menenti, M., Marani, M., 2009. Retrieval of small-relief marsh morphology from Terrestrial Laser Scanner, optimal spatial filtering, and laser return intensity. Geomorphology, 113, pp. 12-20.

Jensen, J.R., 2007. Remote Sensing of the Environment, 2nd ed. Prentice Hall, Upper Saddle River, NJ.

Kashani, A.G., Olsen, M.J., Parrish, C.E., Wilson, N., 2015. A Review of LIDAR Radiometric Processing: From Ad Hoc Intensity Correction to Rigorous Radiometric Calibration. Sensors, 15. pp. 28099-28128.

Leica Geoystems, 2015. Datasheet Leica ScanStation P40 http://www.leica-geosystems.com/downloads123/hds/ hds/general/brochures-datasheet/Leica_ScanStation_P30P40_Plant_DS_en.pdf

Leser, H., Stäblein, G., 1975. Geomorphologische Kartierung, 2nd ed. Berliner Geographische Abhandlungen, Berlin.

Nex, F., Rinaudo, F., 2011. LiDAR or Photogrammetry? Integration is the answer. Ital. J. Remote Sens, 43, pp. 107-121.

Ouédraogo, M.M., Degré, A., Debouche, C., Lisein, J., 2014. The evaluation of unmanned aerial system-based photogrammetry and terrestrial laser scanning to generate DEMs of agricultural watersheds. Geomorphology, 214, pp. 339-355.

Riegl LMS GmbH, 2015. Datasheet Riegl VZ-2000 http://www.riegl.com/uploads/tx_pxpriegldownloads/DataSheet _VZ-2000_2015-03-24.pdf

Riegl LMS GmbH, 2010. Datasheet Riegl LMS-Z420i http://www.riegl.com/uploads/tx_pxpriegldownloads/10_DataS heet_Z420i_03-05-2010.pdf
Shahbazi, M., Sohn, G., Théau, J., Menard, P., 2015. Development and Evaluation of a UAV-Photogrammetry System for Precise 3D Environmental Modeling. Sensors, 15, pp. 27493 27524.

Smith, M.W., Carrivick, J.L., Quincey, D.J., 2015. Structure from motion photogrammetry in physical geography. Prog. Phys. Geogr, pp. 1-29.

Tarolli, P., 2014. High-resolution topography for understanding Earth surface processes: Opportunities and challenges. Geomorphology 216, 295-312. Tong, X., Liu, X., Chen, P., Liu, S., Luan, K., Li, L., Liu, S., Liu, X., Xie, H., Jin, Y., Hong, Z., 2015. Integration of UAV-Based Photogrammetry and Terrestrial Laser Scanning for the Three-Dimensional Mapping and Monitoring of Open-Pit Mine Areas. Remote Sens, 7, pp. 6635-6662.

Topcon Positioning Systems, I., 2006. HiPer Pro Operator's Manual http://www.top-survey.com/top-survey/downloads/ HiPerPro_om.pdf

Turner, D., Lucieer, A., Watson, C., 2012. An automated technique for generating georectified mosaics from ultra-high resolution Unmanned Aerial Vehicle (UAV) imagery, based on Structure from Motion (SFM) point clouds. Remote Sens, 4, pp. $1392-1410$.

Velodyne, 2014. Velodyne HDL-64E User's Manual http://www.velodynelidar.com/lidar/products/manual/63HDL64E S2 Manual_Rev D_2011_web.pdf

Westoby, M.J., Brasington, J., Glasser, N.F., Hambrey, M.J., Reynolds, J.M., 2012. "Structure-from-Motion" photogrammetry: A low-cost, effective tool for geoscience applications. Geomorphology, 179, pp. 300-314.

Zepp, H., 2014. Geomorphologie, 6th ed. UTB, Stuttgart. 